EESTI NSV TEADUSTE AKADEEMIA TOIMETISED. XIII KOIDE FOUSIKA-MATEMAATIKA- JA TEHNIKATEADUSTE SEERIA. 1964, NR. 3

ИЗВЕСТИЯ АКАДЕМИИ НАУК ЭСТОНСКОИ ССР. ТОМ ХІІІ

СЕРИЯ ФИЗИКО-МАТЕМАТИЧЕСКИХ И ТЕХНИЧЕСКИХ НАУК. 1964, 은 3

\title{
СПЕКТРАЛЬНЫЙ РАДИАЦИОННЫЙ РЕЖИМ ПОСЕВА КУКУРУЗЫ И РАСЧЕТ ФОТОСИНТЕТИЧЕСКИ АКТИВНОЙ РАДИАЦИИ (ФАР)
}

\author{
ХЕРБЕРТ НИИЛИСК
}

В статье излагаются результаты спектрофотометрнческого исследования радиационного поля внутри посева кукурузы во время комплексной экспедиции в Иыгева (Эст. ССР) в 1963 г. Получены средние горизонтальные спектральные потоки радиации на разных уровнях в посеве при пяти длинах волны. На основе этих данных рассчитано спектральное поглощение посева по слоям. Предложена методика расчета фотосинтетически активной радиации (ФАР) по спектральным измерениям. Рассчитана ФАР внутри посева и приведены некоторые примеры расчета фотосинтеза посева.

\section{1. Спектральные потоки радиации в посеве}

На основе большого количества измерений оптических свойств листьев растений $\left[{ }^{1-9}\right]$ известно, что эти свойства, т. е. отражение; пропускание и поглощение имеют селективный характер. Поэтому можно предполагать, что посев в целом, как оптическая система, имеет тақже селективные свойства по отношению пропускания, отражения и поглощения. Из этого вытекает, что для детального изучения радиационного режима посевов нужно провести измерения по отдельным длинам волн, т. е. измерить спектральные потоки радиации. До сих пор данных о спектральных потоках радиации внутри растительности весьма мало. Имеются лишь некоторые данные Сауберера [10] в низкой растительности и ряд измерений под пологом леса [11-14]. Несколько больше таких измерений, при которых пытаются выделить селективными приемниками и разными фильтрами фотосинтетически активную область спектра $(\Phi A P)$.

Теоретические расчеты потоков радиации внутри посева [15-18] обычно также проводятся для монохроматических потоков. Поэтому для проверки теории необходимо знать данные о спектральных потоках.

В составе комплексной экспедиции по изучению фотосинтеза и радиационного режима посевов сельскохозяйственных культур (летом 1963 г. в Кыгева) автором были проведены измерения следуюгих спектральных потоков радиации внутри рядового посева кукурузы (расстояние между рядами $60 \mathrm{~cm}$, сорт Буковина 3) при пяти длинах волны:

1) Нисходящие потоки радиации на высотах $40 \div 200$ см от поверхности земли через каждые 20 см. При этом в зависимости от высоты посева часть высот выпадала.

2) Восходящие потоки радиации на высотах $32 \div 172$ см через каждые 20 см. Здесь также в зависимости от высоты посева часть высот выпадала. 
Длины волны были выбраны по кривой отражения зеленого листа с таким расчетом, что они приближенно попали в характерные точки этой кривой [19], а именно: $400,490,552,676$ и 764 mu. Измерения проводили интегрирующим спектрофотометром конструкции автора [20]. Одновременно с измерениями внутри посева измерялась регистрирующим спектрофотометром [2'] попадающая пасев суммарная радиация при тех же длинах волны. Это было необходимо для исключения влияния изменения падающей радиации, а также для получения необходимых сведений о спектральном составе падающей радиации.

По падающему на посев потоку радиации $Q_{0}(\lambda)$ и потоку внутри посева $Q(z, \lambda)$ определяется пропускание слоя посева от уровня $z$ до $z_{0}$

$$
\alpha_{Q}(z, \hat{\lambda})=\frac{Q(z, \hat{\lambda})}{Q_{0}(\lambda)} .
$$

Таким же образом восходящие на разных высотах потоки были выра жены в относительных единицах по отношению к падающей радиациц, так как этим в некоторой мере исключается влияние изменения падающей радиации во время измерения. Обозначим эту величину

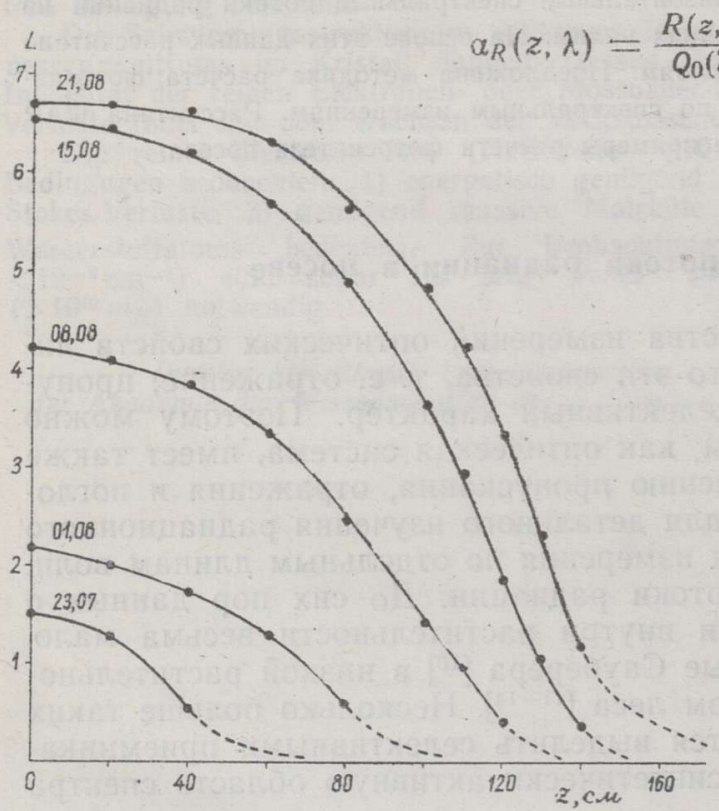

Рис. 1. Функция $L(z)$ для некоторых дней вегетационного периода.

где $R(z, \lambda)$ восходящий поток радиации на уровне $z$. Имея в виду обобщение резуль татов, выразить $\alpha Q$ и $\alpha_{R}$ в зависимости от $z$ нецелесообразно, так как основные элементы посева, ослабляющие радиацию - листья растения - распределяются неравномерно по высоте. Поэтому вместо $z$ нужно найти характеристику посева, как оптической среды, которая лучше определяет оптическую плот ность посева. Мы выбрали вместо аргумента $z$ п.тощадь тех листьев $L(z)$, которые находятся выше данного уровня z. Такие данные были получены прямыми измерениями площади листьев по 10-сантиметровым слоям. Измерения площади производились на фотопланиметре. Зависимость $L$ от $z$ для некоторых дней в течение вегетационного периода изображена на рис. 1 .

Выражая измеренные в посеве величины $\alpha_{Q}$ и $\alpha_{R}$ в зависимости о $L$, мы в значительной степени можем исключить влияние высоты и густоты посева и применять полученные результаты в некотором приближении для посевов разной высоты.

Чувствительность измерительной аппаратуры можно было изменять в широких пределах, поэтому все потоки радиации, т. е. потоки над растительностью и в глубине посева измерены примерно с одинаковой относительной точностью и сглаживание измеренных величин (которые над посевом и под посевом отличаются более, чем в 100 раз) было целесообразно произвести в логарифмическом масштабе. Зависимость меж- 


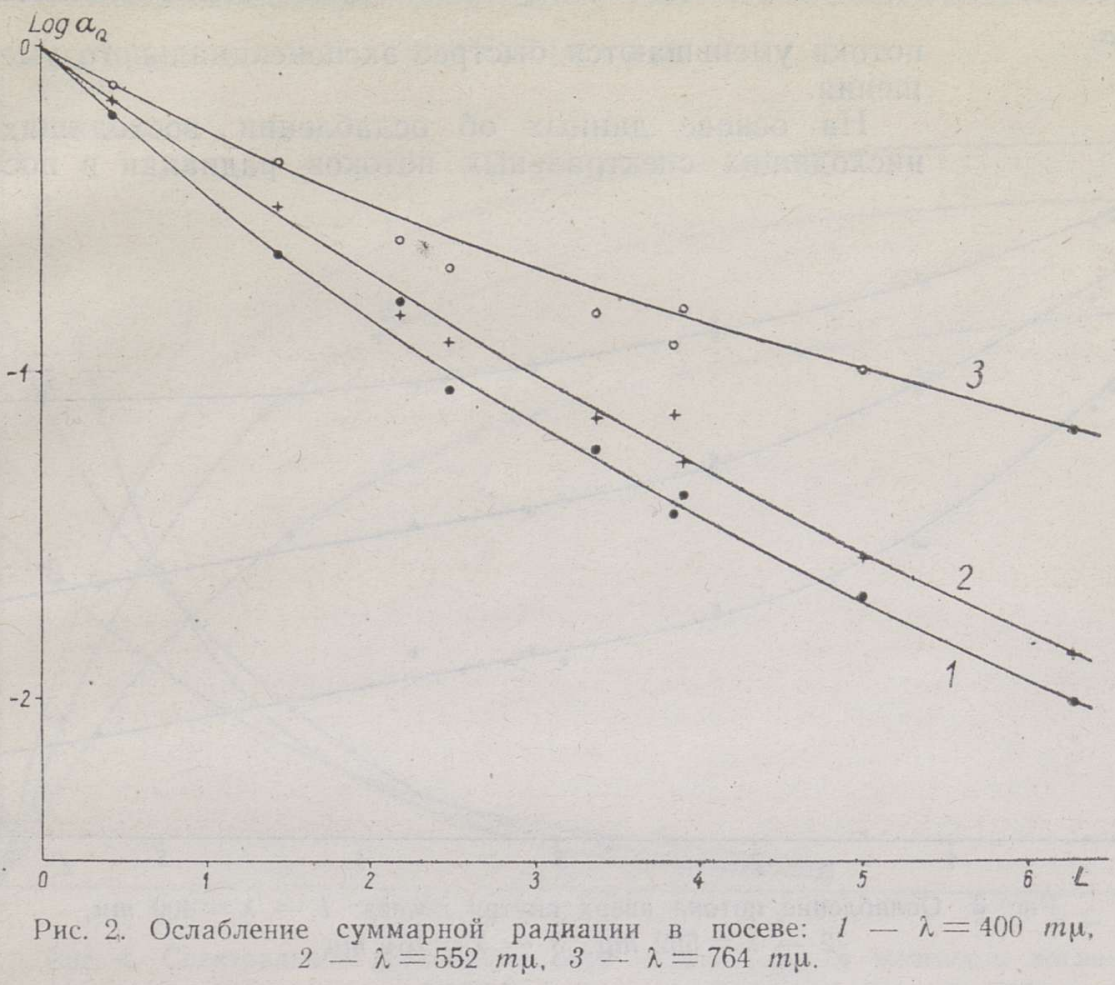

ду $\log \alpha_{Q}$ и $L$ для длин волн $400,552,764$ ти изображена на рнс. 2. Как видно на рисунке, разброс точек измерения в середнне кривой, т. е. при средних $L$, несколько больше, чем при малых и больших $L$. Это получается потому, что там густота листьев большая и в результате этого влиянне измерительной аппаратуры на структуру посева больше.

Все сказанное относится и к кривым $\alpha_{R}$, сглаживание которых также производилось в логарифмическом масштабе. Для длин волн 400 , 552 и $764 m \mu$ точки измерения и сглаженная кривая $\log \alpha_{R}$ приведены на рис. 3 .

По сглаженным в логарифмическом масштабе зависимостям $\log \alpha_{\varrho}$ и $\log \alpha_{R}$ от $L$ получены соответствующие зависимости $\alpha_{Q}$ и $\alpha_{R}$ от $L$. Средние значения $\alpha_{Q}$ и $\alpha_{R}$ для ясного дня при высоте Солнца $30 \div 50^{\circ}$ и при условии, что направление рядов посева. не совпадает с направлением азимута Солнца, приведены в табл. 1 и 2.

Как и можно было ожидать, ослабление радиации в посеве имеет - селективный характер. Второй вывод, вытекающий из этих данных, следующий: ослабление падающих потоков радиации внутри посева в зависимости от $L$ имеет в первом приближении экспоненциальный характер, а восходящие

Таблий 1

\begin{tabular}{l||c|c|c|c|c}
\hline \multicolumn{6}{|c}{${ }^{\alpha} Q$} \\
\hline
\end{tabular}




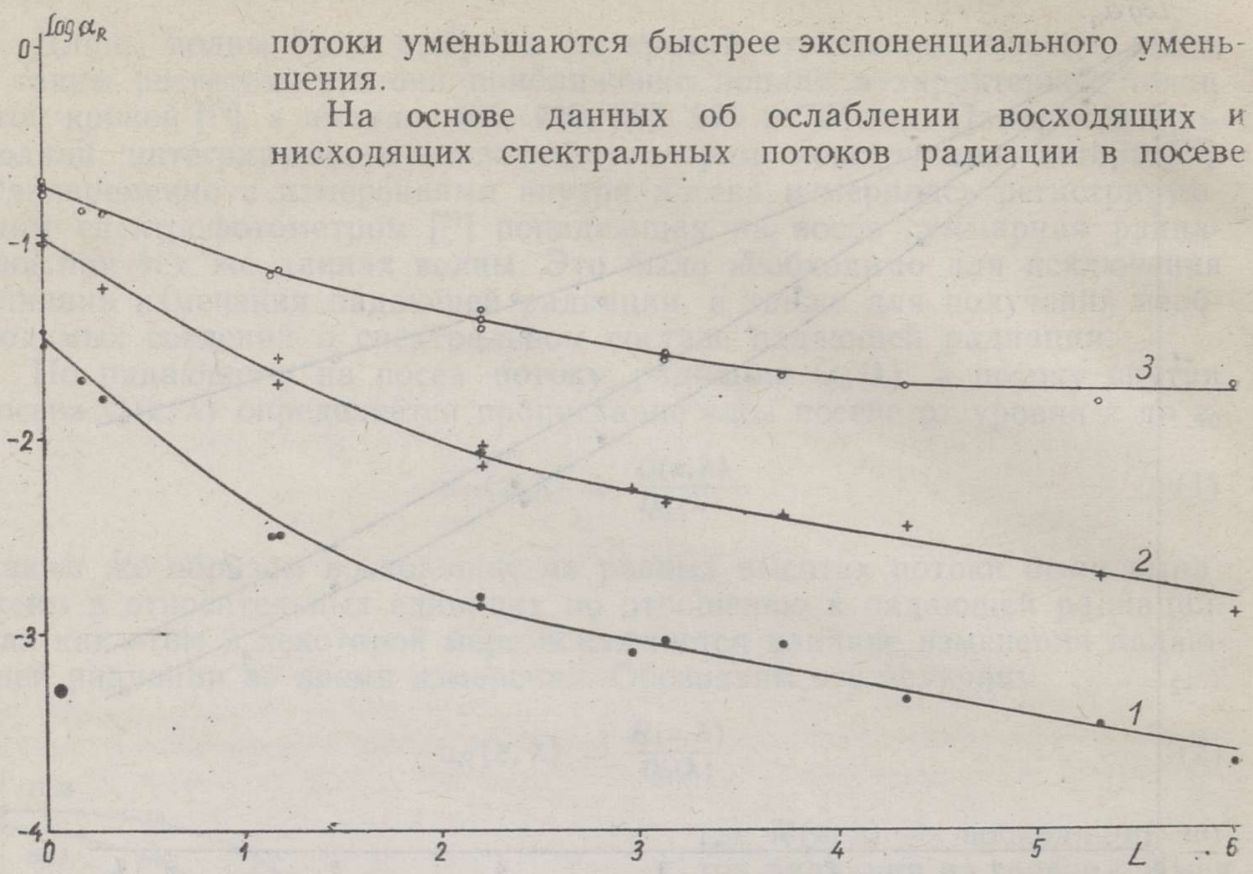

Рис. 3. Ослабленне потока вверх внутри посева: $1-\lambda=400 m \mu$,

$$
2-\lambda=552 m \mu, 3-\lambda=764 m \mu
$$

можно рассчитать поглощенные посевом спектральные потоки П $(\lambda, L)$. Рассмотрим слой посева от верхней границы до уровня $L$, т. е. до уровня, выше которого остается относительная площадь листьев L. Этот слой поглощает радиацию

$$
\begin{gathered}
\Pi(\lambda, L)=Q_{0}(\lambda)-Q(\lambda, L)-\left[R_{0}(\lambda)-R(\lambda, L)\right]= \\
=Q_{0}(\lambda)\left[1-A(\lambda)-\alpha_{Q}(\lambda, L)+\alpha_{R}(\lambda, L)\right],
\end{gathered}
$$

где $A(\lambda)$ - спектральное альбедо.

Относительное поглощение $\alpha_{\pi}(\lambda, L)$ равно

$$
a_{\Pi}(\lambda, L)=\frac{\Pi(\lambda, L)}{Q_{0}(\lambda)}=1-A-\left(\alpha_{Q}-\alpha_{k}\right)
$$

\begin{tabular}{|c|c|c|c|c|c|}
\hline \multicolumn{6}{|c|}{$\boldsymbol{\alpha}_{R}$} \\
\hline & 400 & 490 & 552 & 676 & 764 \\
\hline 0,0 & 0,0343 & 0,0592 & 0,102 & 0,0468 & 0,200 \\
\hline 0,5 & 0,0107 & 0,0232 & 0,0473 & 0,0212 & 0,123 \\
\hline 1,0 & 0,00461 & 0,0106 & 0,0255 & 0,0108 & 0,0822 \\
\hline 1,5 & 0,00254 & 0,00553 & 0,0151 & 0,00621 & 0,0589 \\
\hline 2,0 & 0,00166 & 0,00321 & 0,00977 & 0,00392 & 0,0447 \\
\hline 2,5 & 0,00122 & 0,00205 & 0,00679 & 0,00267 & 0,0351 \\
\hline 3,0 & 0,00092 & 0,00142 & 0,00501 & 0,00193 & 0,0283 \\
\hline 3,5 & 0,00072 & 0,00107 & 0,00386 & 0,00148 & 0,0238 \\
\hline 4,0 & 0,00056 & 0,00084 & 0,00308 & 0,00119 & 0,0208 \\
\hline 4,5 & 0,00045 & 0,00069 & 0,00249 & 0,00100 & 0,0187 \\
\hline 5,0 & 0,00036 & 0,00058 & 0.00212 & 0,00087 & 0,0174 \\
\hline 5,5 & 0,00029 & 0,00049 & 0,00186 & 0,00077 & 0,0166 \\
\hline 6,0 & 0,00023 & 0,00042 & 0,00164 & 0,00069 & 0,0164 \\
\hline
\end{tabular}

Таблица 2 


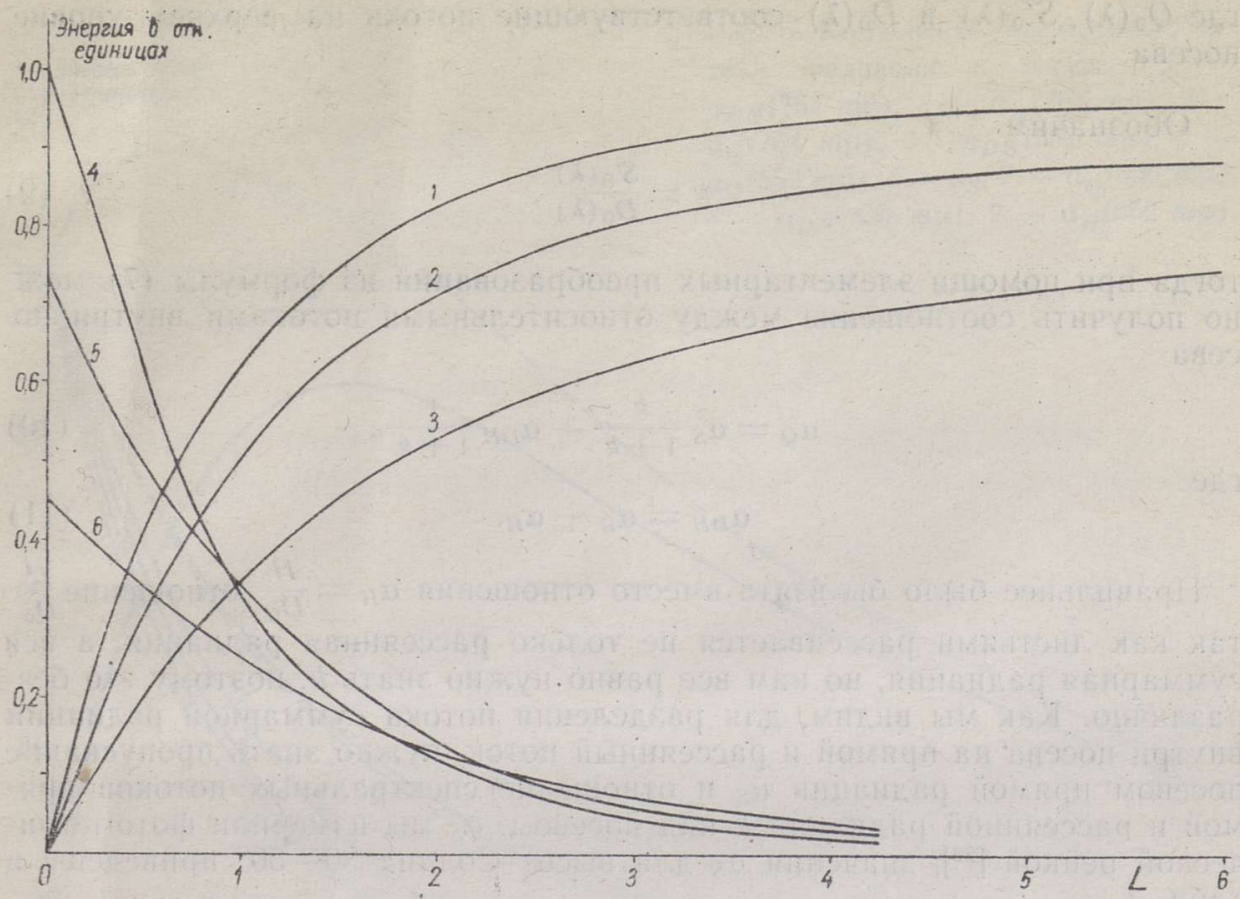

Рис. 4. Спектральное поглощение слоя посева $\left(\alpha_{\Pi}\right)$ и плотность поглощения $\left(\partial \alpha_{\Pi} / \partial L\right): 1-\alpha_{\Pi}(400 m \mu), 2-\alpha_{\Pi}(552 m \mu), 3-\alpha_{\Pi}(764 m \mu)$; $4,5,6$ - соответствующие плотности поглощения.

Кривые $\alpha_{n}$ для длин волн 400,552 и $764 m \mu$ в зависимости от $L$ даны на рис. 4. При больших $L$

$$
\lim _{n} \alpha_{n}=1-A \text {. }
$$

Для дальнейших расчетов нужно знать плотность поглощения на данном уровне, т. е. сколько поглощает слой на уровне, имеющий относительную площадь листьев, равную единице. Әта величина получается из (4) путем дифференцирования по $L$

$$
\frac{\partial \alpha_{\Pi}}{\partial L}=-\left(\frac{\partial \alpha_{Q}}{\partial L}-\frac{\partial \alpha_{R}}{\partial L}\right)
$$

Кривые $\partial \alpha_{n} / \partial L$ изображены на рис. 4.

Спектральный поток суммарной радиации внутри посева состоит из следующих компонентов:

$$
Q(\lambda, L)=S^{\prime}(\lambda, L)+D(\lambda, L)+H(\lambda, L),
$$

где $S^{\prime}(\lambda, L)$ - поток прямой радиации на уровне $L$,

$D(\lambda, L)$ - поток рассеянной раднации неба на уровне $L$,

$H(\lambda, L)$ - поток рассеянной от листьев суммарной радиации на уровне $L$.

Разделяем относительный поток суммарной радиации на отдельные компоненты. Рассмотрим опять относительные величины

$$
\alpha_{Q}=\frac{Q(\lambda, L)}{Q_{0}(\lambda)}, \quad \alpha_{S}=\frac{S^{\prime}(\lambda, L)}{S_{0}^{\prime}(\lambda)}, \quad \alpha_{D}=\frac{D(\lambda, L)}{D_{0}(\lambda)}, \quad \alpha_{H}=\frac{H(\lambda, L)}{D_{0}(\lambda)},
$$


где $Q_{0}(\lambda), S_{0}^{\prime}(\lambda)$ и $D_{0}(\lambda)$ соответствуюцие потоки на верхнем уровне посева.

Обозначнм

$$
k=\frac{S_{0}^{\prime}(\lambda)}{D_{0}(\lambda)}
$$

тогда при помощн элементарных преобразований нз формулы (7) можно получить соотношенне нежду относнтельными потоками внутри посева

$$
\alpha_{Q}=\alpha_{S} \frac{k}{1+k}+\alpha_{1 \lambda H} \frac{1}{1+k}
$$

где

$$
\alpha_{D H}=\alpha_{D}+\alpha_{H}
$$

Правнльнее было бы взять вместо отношения $\alpha_{H}=\frac{H}{D_{0}}$ отношение $\frac{H}{Q_{0}}$, так как листьями рассеивается не только рассеянная радиация, а вся суммарная радиация, но нам все равно.нужно знать $k$, поэтому это безразлично. Қак мы видим, для разделения потока суммарной радиации внутри посева на прямой и рассеянный поток, нужно знать пропускание посевом прямой радиации $\alpha_{S}$ и отношение спектральных потоков прямой и рассеянной радиации $k$ над посевом. $\alpha_{S}$ мы нзмерили фотометрической рейкой $\left[{ }^{22}\right]$, значения $\alpha_{S}$ для высот Солнца $30-50^{\circ}$ приведены в табл. 3.

Таблица зे

\begin{tabular}{c||c|c|c|c|c|c|c|c}
\hline$L$ & 0 & 0,5 & 1,0 & 1,5 & 2,0 & 3,0 & 4,0 & 6,0 \\
\hline$\alpha_{S}$ & 1,000 & 0,606 & 0,368 & 0,223 & 0,135 & 0,050 & 0,018 & 0,002 \\
\hline
\end{tabular}

К сожалению, во время экспедиции не было измерено отношение $k$. Для этого нужно было бы измерить раздельно спектральные потоки прямой и рассеянной радиаций. Пока придется использоваться опубликованными данными литературы $\left[{ }^{23,24]}\right.$. Мы использовались данными работ О. Авасте и др. $\left[{ }^{23}\right]$, где теоретически рассчитаны спектральные потоки прямой и рассеянной радиаций для ясной погоды при оптической толшине $\tau_{0}=0,3$ и массе атмосферы $m=2\left(h_{\odot}=30^{\circ}\right)$. Эти условия близки к нашим условиям измерения. Хотя применение такого теоретически найденного отношения довольно приближенное, можно все-таки провести ориентировочные расчеты. В результате мы получим относительное ослабление рассеянной радиации в посеве. Кривые $\alpha_{Q}, \alpha_{S}$ и $\alpha_{D H}$ для трех длин волн показаны на рис. 5. Мы видим, что при 400 mu суммарный, прямой и рассеянный потоки ослабляются внутри посева примерно одинаково. Измерения коэффициентов пропускания и отражения листьев кукурузы показывают, что отражение при $400 m \mu$ около $4-5 \%$, а пропускание $2 \%$, т. е. лист поглощает около $93 \div 94 \%$ от всей падающей на него радиации. Таким образом, для радиации при $\lambda=400$ ми листья почти черные, и основная часть потока радиации внутри посева приходит от неба. Обозначим среднюю прозрачность растительности в направлении $(\vartheta, \varphi)$ на уровне $L$ внутри посева через $\alpha(\vartheta, \varphi, L)$ и интенсивность радиации от неба $I_{D}(\vartheta, \varphi, \lambda)$. Тогда спектральный поток радиации, приходящий на уровень $L$ от неба, равен 


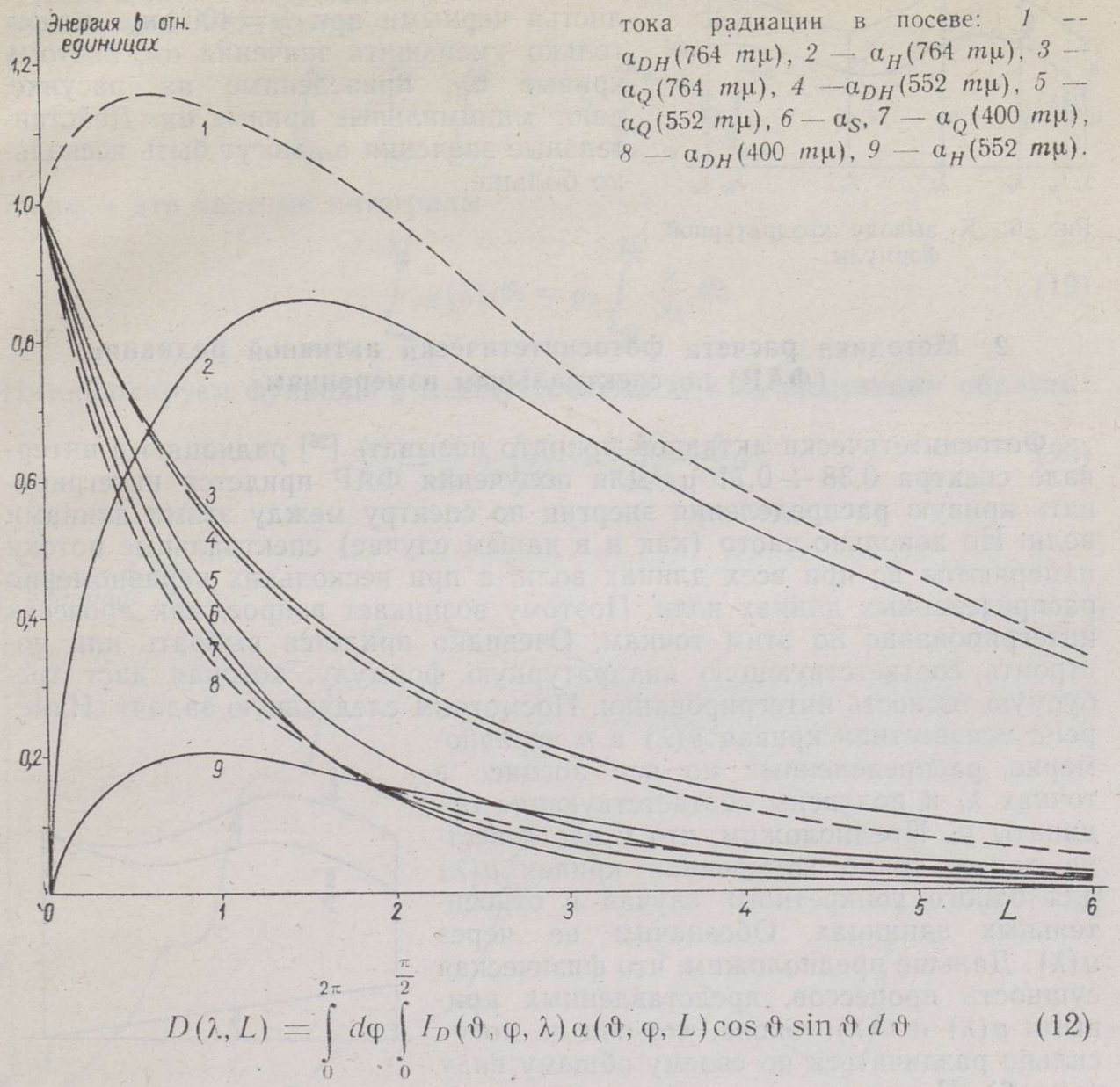

Рнс. 5. Компоненты нисходяшего потока радиацин в посеве: 1 -$\alpha_{D H}(764 m \mu), 2-\alpha_{H}(764 m \mu), 3-$ $\alpha_{0}(764 m \mu), 4-\alpha_{D H}(552 m \mu), 5-$ $\alpha_{Q}(552 m \mu), 6-\alpha_{S}, 7-\alpha_{Q}(400 m \mu)$, $8-\alpha_{D H}(400 m \mu), 9-\alpha_{H}(552 m \mu)$.

на уровне $L=0$.

$$
D_{0}(\lambda)=\int_{0}^{2 \pi} d \varphi \int_{0}^{\frac{\pi}{2}} I_{D}(\vartheta, \varphi, \lambda) \cos \vartheta \sin \vartheta d \vartheta
$$

отсюда

$$
a_{D}=\frac{D(\lambda, L)}{D_{0}(\lambda)}=\bar{\alpha}(L)
$$

где $\bar{\alpha}(L)$ - некоторое среднее значение $\alpha$, независящее от $\lambda$. Отсюда видно, что $\alpha_{D}$ не зависит от длины волны.

При $\lambda=400 m \mu \alpha_{H} \ll \alpha_{D}$ и

$$
\alpha_{D H} \approx \alpha_{D}=\bar{\alpha}(L) \text {. }
$$

Используя это обстоятельство, мы можем для других длин волн найти $\varkappa_{H}$, т. е. долю рассеянной радиации внутри посева, рассеиваемую листьями растений. Соответствующие зависимости $\alpha_{H}$ от $L$ приведены на рис. 5. Видно, что инфракрасная часть рассенвается гораздо силььнее, чем ФАР. На уровне $L \approx 2$ прн $\lambda=764$ ми поток $H(\lambda, L)$ примерно ра. вен потоку $D_{0}(\lambda)$ от неба. 


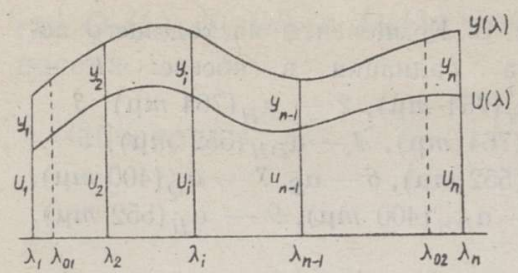

Рис. 6. К выводу квадратурной формулы.
Ошибка, которую мы сделаем, считая листья черными при $\lambda=400 \mathrm{m \mu}$, может только уменьшить значения $\alpha_{H}$, поэтому кривые $\alpha_{H}$, приведенные на рисунке, дают минимальные кривые $\alpha_{H}$. Действительные значения $\alpha_{H}$ могут быть несколько больше.

\section{2. Методика расчета фотосинтетически активной радиации (ФАР) по спектральным измерениям}

Фотосинтетически активной принято называть $\left[{ }^{25}\right]$ радиацию в интервале спектра $0,38 \div 0,71 \mu$. Для получения ФАР придется интегрировать кривую распределения энергии по спектру между этими длинами волн. Но довольно часто (как и в нашем случае) спектральные потоки измеряются не при всех длинах волн, а при нескольких неравномерно распределенных длинах волн. Поэтому возникает вопрос, как провести интегрирование по этим точкам. Очевидно придется выбрать или построить соответствующую квадратурную формулу, которая даст требуемую точность интегрирования. Посмотрим следующую задачу. Измерена неизвестная кривая $y(\lambda)$ в $n$ неравномерно распределенных по оси абсцисс в точках $\lambda_{i}$ и получены соответствующие ординаты $y_{i}$. Предположим, что у нас известна также точно измеренная кривая $y(\lambda)$ для одного конкретного случая в относительных единицах. Обозначим ее через $u(\lambda)$. Дальше предположим, что физическая сущность процессов, представленных кривыми $y(\lambda)$ и $u(\lambda)$ такова, что они не могут сильно различаться по своему общему виду (рис. 6). Например, если говорить конкретно об изменении спектрального состава

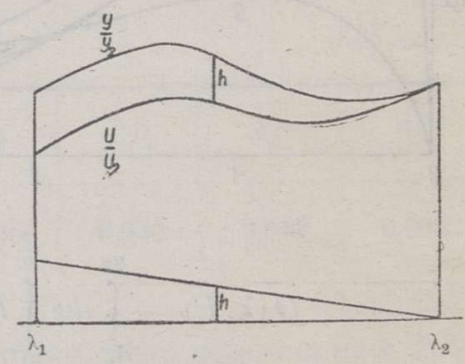

Рис. 7. К выводу формулы (20). суммарной радиации ясной погоды, то этот состав сильно не изменяется. Максимум кривой распределения энергии в спектре всегда находится около $500 \mathrm{~m} \mu$; в сторону длинных волн кривая равномерно падает, а в сторону коротких волн имеются характерные полосы поглощения. Спектральный состав отраженной от растительности радиации также имеет характерный вид с полосой поглоще-

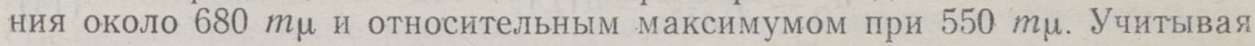
приведенные предположения, постараемся построить возможно точную жвадратурную формулу для вычисления интеграла

$$
\int_{\lambda_{01}}^{\lambda_{02}} u(\lambda) d \lambda
$$

Общий вид всякой квадратурной формулы следующий:

$$
\int_{\lambda_{01}}^{\lambda_{02}} y(\lambda) d \lambda=\sum_{i=1}^{n} a_{i} y_{i}
$$


Нужно найти коэффициенты $a_{i}$. Для этого представим интеграл (16) в виде суммы интегралов

$$
\int_{\lambda_{01}}^{\lambda_{02}}=\int_{\lambda_{01}}^{\lambda_{2}}+\int_{\lambda_{2}}^{\lambda_{3}}+\ldots+\int_{\lambda_{n-3}}^{\lambda_{n-1}}+\int_{\lambda_{n-1}}^{\lambda_{02}} .
$$

Найдем эти частные интегралы

$$
\int_{\lambda_{01}}^{\lambda_{2}} y(\lambda) d \lambda=y_{2} \cdot \int_{\lambda_{01}}^{\lambda_{2}} \frac{y}{y_{2}} d \lambda
$$

Интерполируем функцию $y$ между точками $\lambda_{1}$ и $\lambda_{2}$ следующим образом:

$$
\frac{y}{y_{2}}=\frac{u}{u_{2}}+\left(\frac{y_{1}}{y_{2}}-\frac{u_{1}}{u_{2}}\right) \frac{\lambda_{2}-\lambda}{\lambda_{2}-\lambda_{1}} \text {. }
$$

Энергия В отн.

eguнuцах

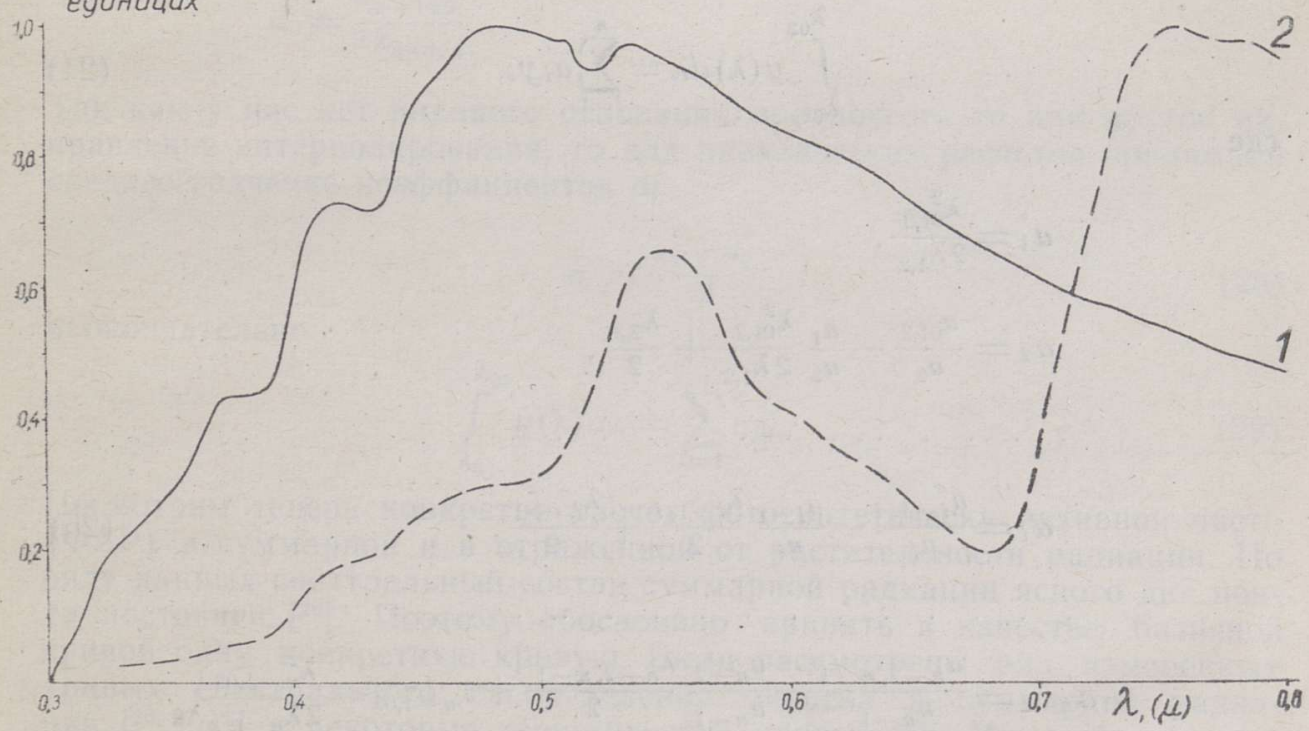

Рис. 8. Распределение энергии в спектре суммарной (1) и отраженной. (2) радиации.

Это значит, что мы интерполируем разность известной функции $u / u_{2}$ и неизвестной функции $y / y_{2}$ между точками $\lambda_{1}$ и $\lambda_{2}$ линейно, как показано на рис. 7. Используя формулу (20), легко провести интегрирование, при этом для краткости обозначим

$$
\begin{array}{r}
\int_{\lambda_{i}}^{\lambda_{i+1}} u d \lambda=u_{i, i+1}, \quad \lambda_{i+1}-\lambda_{i}=\lambda_{i, i+1}, \\
\int_{\lambda_{01}}^{\lambda_{2}} y(\lambda) d \lambda=\frac{\lambda_{01,2}^{2}}{2 \lambda_{1,2}} y_{1}+\left(\frac{u_{01,2}}{u_{2}}-\frac{u_{1}}{u_{2}} \frac{\lambda_{01,2}^{3}}{2 \lambda_{1,2}}\right) y_{2}
\end{array}
$$


Совершенно аналогично получнм следующие интегралы:

$$
\begin{aligned}
\int_{\lambda_{2}}^{\lambda_{3}} y(\lambda) d \lambda & =\frac{\lambda_{2,3}}{2} y_{2}+\left(\frac{u_{2,3}}{u_{3}}-\frac{u_{2}}{u_{3}} \frac{\lambda_{2,3}}{2}\right) y_{3,} \\
\int_{\lambda_{i}}^{\lambda_{i+1}} y(\lambda) d \lambda & =\frac{\lambda_{i, i+1}}{2} y_{i}+\left(\frac{u_{i, i+1}}{u_{i+1}}-\frac{u_{i}}{u_{i+1}} \frac{\lambda_{i, i+1}}{2}\right) y_{i+1}, \\
\int_{\lambda_{n-1}}^{\lambda_{02}} y(\lambda) d \lambda & =\left(\lambda_{n-1,02}-\frac{\lambda_{n-1,02}^{2}}{2 \lambda_{n-1, n}}\right) y_{n-1}+\left[\frac{u_{n-1,02}}{u_{n}}-\right. \\
& \left.-\frac{u_{n-1}}{u_{n}}\left(\lambda_{n-1,02}-\frac{\lambda_{n-1,02}^{2}}{2 \lambda_{n-1, n}}\right)\right] y_{n} .
\end{aligned}
$$

Суммируя интегралы (22) и (23), получим

rде

$$
\int_{\lambda_{01}}^{\lambda_{02}} y(\lambda) d \lambda=\sum_{i=1}^{n} a_{i} y_{i}
$$

$$
\begin{aligned}
& a_{1}^{\prime}=\frac{\lambda_{01,2}^{2}}{2 \lambda_{1,2}} \\
& a_{2}^{\prime}=\frac{u_{01,2}}{u_{2}}-\frac{u_{1}}{u_{2}} \frac{\lambda_{01,2}^{2}}{2 \lambda_{1,2}}+\frac{\lambda_{2,3}}{2}, \\
& a_{i}^{\prime}=\frac{u_{i-1, i}}{u_{i}}-\frac{u_{i-1}}{u_{i}} \frac{\lambda_{i-1, i}}{2}+\frac{\lambda_{i, i+1}}{2} \\
& a_{n-1}^{\prime}=\frac{u_{n-2, n-1}}{u_{n-1}}-\frac{u_{n-2}}{u_{n-1}} \frac{\lambda_{n-2, n-1}}{2}+\lambda_{n-1,02}-\frac{\lambda_{n-1,02}^{2}}{2 \lambda_{n-1, n}}, \\
& a_{n}=\frac{u_{n-1,02}}{u_{n}}-\frac{u_{n-1}}{u_{n}}\left(\lambda_{n-1,02}-\frac{\lambda_{n-1,02}^{2}}{2 \lambda_{n-1, n}}\right)
\end{aligned}
$$

Формулы (25) для коэффициентов $a_{i}^{\prime}$ одинаковы как для $\lambda_{01}>\lambda_{1}$, так и для $\lambda_{01}<\lambda_{1}$.

При интерполяции разности функции $y / y_{2}$ и $u / u_{2}$ мы интерполировали ее «назад», т. е. начинали интерполяцию от правой ординаты промежутка (рис. 7). Таким же образом можно начинать интерполяцию с левого ордината и получить коэффициенты квадратурной формулы при интерполировании «вперед». Тогда

$$
\int_{\lambda_{01}}^{\lambda_{02}} y(\lambda) d \lambda=\sum_{i=1}^{n} a_{i}^{\prime \prime} y_{i},
$$


где

$$
\begin{aligned}
a_{1}^{\prime \prime} & =\frac{u_{01,2}}{u_{1}}-\frac{u_{2}}{u_{1}}\left(\lambda_{01,2}-\frac{\lambda_{01,2}^{2}}{2 \lambda_{1,2}}\right) \\
a_{2} & =\frac{u_{2,3}}{u_{2}}-\frac{u_{3}}{u_{2}} \frac{\lambda_{2,3}}{2}+\lambda_{01,2}-\frac{\lambda_{v 1,2}^{2}}{2 \lambda_{1,2}} \cdot \\
a_{3}^{\prime \prime} & =\frac{u_{3,4}}{u_{3}}-\frac{u_{4}}{u_{3}} \frac{\lambda_{3,4}}{2}+\frac{\lambda_{u_{2}, 3}}{2}, \\
& \cdot \cdot \cdot \frac{u_{i, i+1}^{\prime \prime}}{a_{i}}=\frac{u_{i+1}}{u_{i}} \frac{\lambda_{i, i+1}}{2}+\frac{\lambda_{i-1, i}}{2}, \\
a_{n-1}^{\prime \prime} & =\frac{u_{n-1,02}}{u_{n-1}} \cdot \frac{u_{n}}{u_{n-1}} \frac{\lambda_{n-1,02}^{2}}{2 \lambda_{n-1, n}}+\frac{\lambda_{n-2, n-1}}{2} \\
a_{n}^{\prime \prime} & =\frac{\lambda_{n-1,02}^{2}}{2 \lambda_{n-1, n}} \cdot
\end{aligned}
$$

Так как у нас нетт никакого основания предпочесть то или другое направление интерполировання, то для практнческих расчетов принимаем среднее значение коэффициентов $a_{i}$

$$
a_{i}=\frac{a_{i}^{\prime}+a_{i}^{\prime \prime}}{2}
$$

и окончательно

$$
\int_{\lambda_{01}}^{\lambda_{02}} y(\lambda) d \lambda=\sum_{i=1}^{n} a_{i} y_{i}
$$

Посмотрим теперь конкретно расчет фотосинтетически активной части (ФАР) в суммарной и в отраженной от растительности радиации. По ряду данных спектральный состав суммарной радиации ясного дня почти постоянен $\left[{ }^{26}\right]$. Поэтому обосновано принять в качестве базисной кривой одну конкретную кривую. Были рассмотрены ряд измеренных крнвых спектрального распределения энергии в суммарной радиации $[26,27,28]$ и некоторые теоретические кривые $\left[{ }^{23}\right]$. Мы выбрали кривую из монографии Кондратьева $\left[{ }^{26}\right]$, которая лучше всего согласовывается с нашими измерениями. Кроме того, эта кривая лучше других экспериментальных кривых согласуется и с теоретическими расчетами. Эта кривая приведена на рис. 8. Кривую спектрального распределения энергии, отраженной от растительности радиации, мы получили следующим образом. За основу принята спектральная кривая коэффициента отражения, измеренная Тоомингом [19]. Эту кривую экстраполировали в сторону ультрафиолетовой части на основе данных Шульгина и др. [8] и в сторону ннфракрасной части на основе данных Дирмхирн [9]. Для приближенного получения спектрального состава отраженной радиа ции при ясной погоде коэффициент отражения умножим на спектральное распределение суммарной радиации. В результате получилась кривая 2 (см. рис. 8). Используя эти кривые и Іформулу (28), получнли коэффициенты квадратурной формулы для примененных длнн волн. По измеренным при четырех длинах волн спектральным потокам суммарной радиации рассчитана ФАР для всех случаев нзмерения внутри посева. 


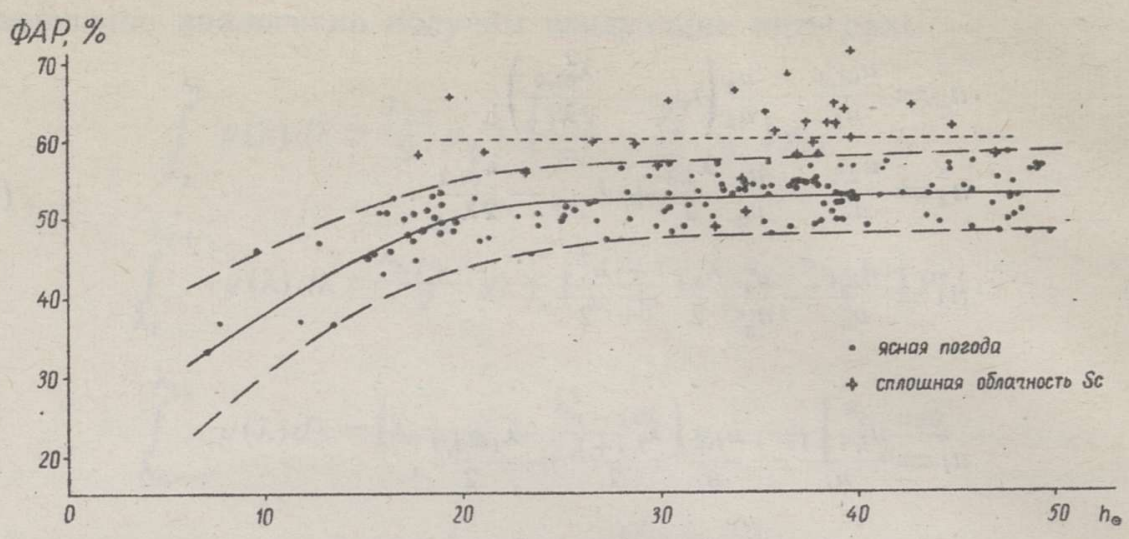

Рис. 9. Процент ФАР в суммарной радиации.

Пиранометром регистрировали суммарную радиацию. Зная ФАР и суммарную радиацию, можно получить процент (или коэффициент) ФАР в суммарной радиации. На рис. 9 показана зависимость коэффициента ФАР от высоты Солнца. На графике нанесены все точки измерения, при незакрытом облаками диска Солнца, полученные в течение экспедиции 1963 г. (июль, август) в Эстонии. На этом же графике показаны точки измерения при сплошной облачности $(S c)$ разной плотности. На рис.9 видно, что коэффициент ФАР для случаев, когда перед диском Солнца нет облаков, составляет в среднем $52,5 \%$, при этом все точки измерения для высот Солнца больше $15^{\circ}$. находятся в промежутке $(52,5 \pm 5) \%$ Для сплошной облачности коэффициент ФАР в среднем $60 \%$, но там колебания величины коэффициента больше. Еще бо́льшие колебания можно получить при других видах облачности, особенно при переменной облачности. Таким же способом можно без всяких затруднений рассчитать ФАР в отраженной от растительности радиации, вычисляя коэффициенты по формуле (28) и используя измерения спектрофотометра над посевом. Несколько сложнее расчет ФАР внутри посева. По ряду данных спектральная кривая пропускания листьев весьма похожа на кривую отражения $\left[{ }^{3,29]}\right.$. Поэтому при вычислении нисходящих потоков $Ф А Р$ на большой глубине в посеве, т. е. при больших $L$, можно использовать коэффициенты квадратурной формулы для отраженной радиа ции, так как там часть приходящей прямо от неба радиации мала. В верхних слоях посева, т. е. при малом $L$ очевидно можно использовать коэффициенты квадратурной формулы для суммарной радиации, так как там, в основном, радиация, приходящая от неба и от Солнца. Самое неясное положение при средних $L$ (около 2). Мы рассчитали для этих $L$ ФАР по обеим коэффициентам. Их разность составлял не больше $5-6 \%$. Такой является и точность расчета для этих $L$. На рис. 10 приведены результаты расчета. Кривая 1 изображает относительное ослабление нисходящего потока ФАР. Кривая 2 показывает восходящий поток ФАР. На основе этих потоков можно рассчитать и поглощенную в слое от 0 до $L$ ФАР, которая дана кривой 3 , а также плотность поглощенная (кривая 4). Зная поглощенную в посеве ФАР и световые кривые фотосинтеза, мы можем рассчитать фотосинтез посева. Но данных о световых кривых кукурузы, особенно в естественных условиях, пока весьма мало $[30,31,32]$. Кроме того, эти кривые нужно знать для листьев разных ярусов. Такие данные пока вообще отсутствуют. Нужно еще отметить, что в ряде случаев измерения фотосинтеза и методика измерения ра- 


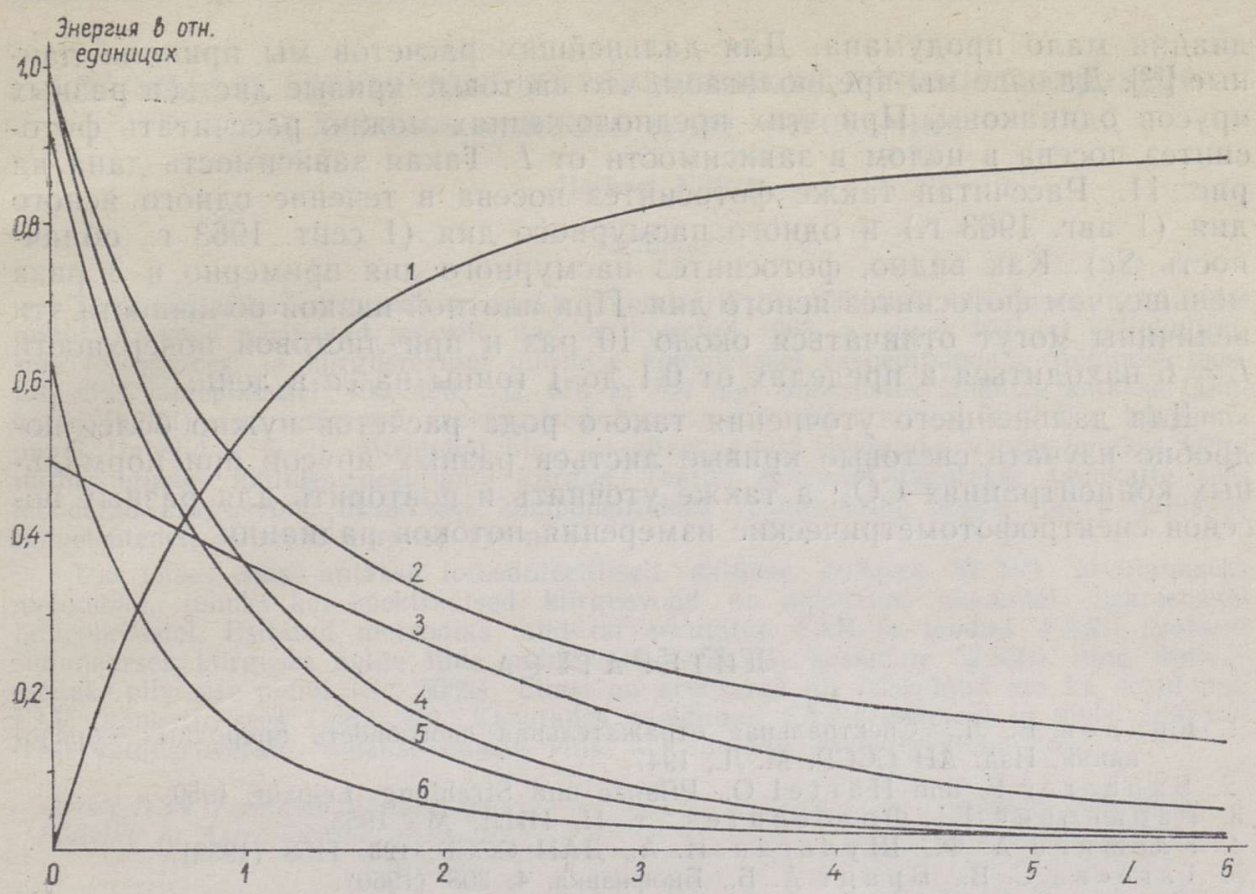

Рис. 10. Относительные потоки ФАР в посеве: 1 - поглощенный в слое $0 \div L$ поток, 2 - ослабление интегральной суммарной радиации, 3 - коэффициент ФАР суммарной радиации в посеве, если над посевом 50\% ФАР, 4 - нисходящий поток, 5 - плотность поглощения на уровне $L, 6$ - восходящий поток (масштаб вертикальной оси в 10 раз увелнчен):

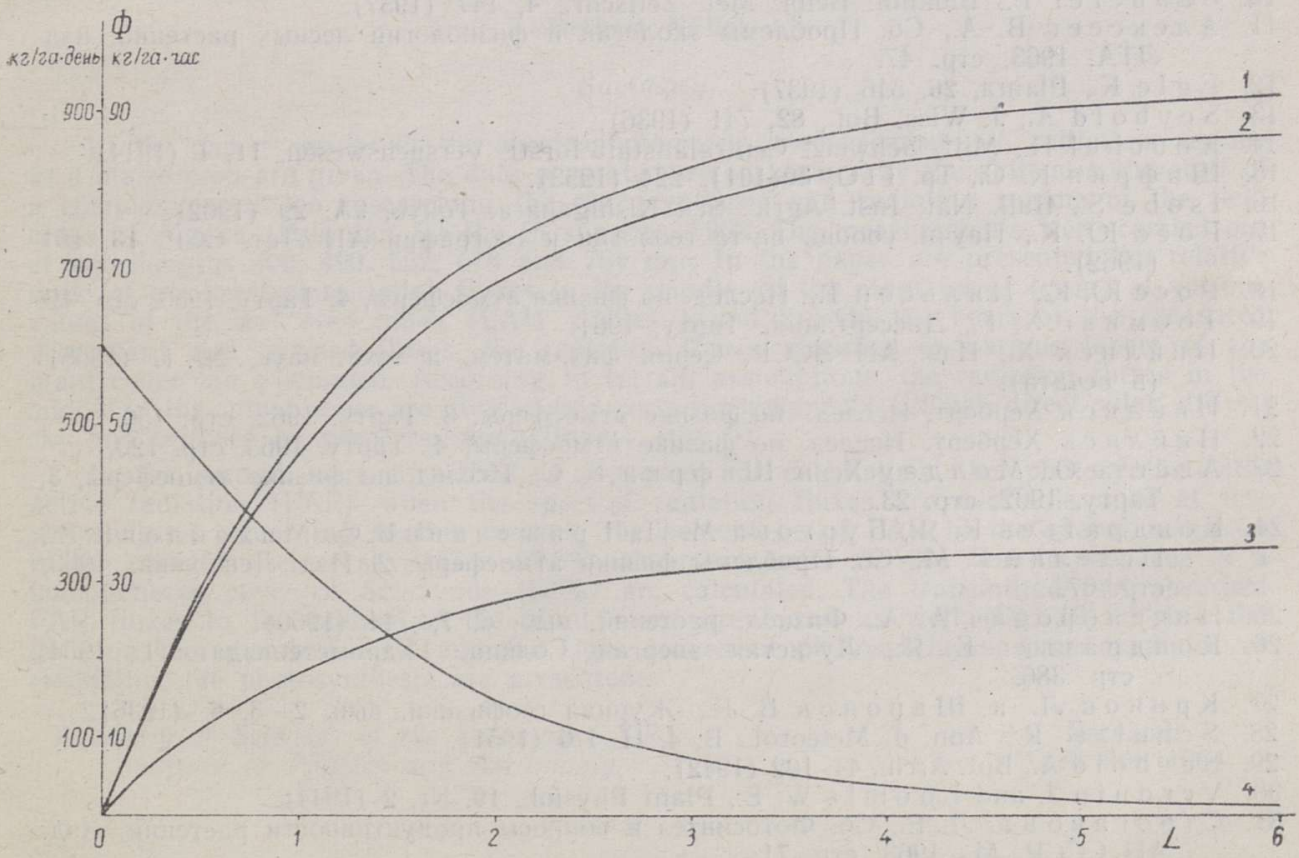

Рнс. 11. Чистый фотосинтез посева: 1 - фотосинтез в ясный день (1 авг. 1963 г.) в завнсимости от $L$ в кг/2a. день, 2 - фотосинтез слоя посева $0 \div L$ в кг/2a. час, 3 фотосинтез в пасмурный день (облачность $S c), 4$ - фотосинтез слоя посева, имеющего относительную плошадь листьев, равную единице. 
диации мало продумана. Для дальнейшнх расчетов мы приняли данные [32]. Далыше мы предполагаем, что световые кривые листьев разных ярусов одннаковы. При этих предположениях можно рассчитать фотосинтез посева в целом в зависимостн от $L$. Такая зависимость дана на рис. 11. Рассчитан также фотосинтез посева в течение одного ясного дня (1 авг. 1963 г.) и одного пасмурного дня (1 сент. 1963 г., облачность $S c)$. Как вндно, фотосинтез пасмурного дня примерно в 3 раза меньше, чем фотосинтез ясного дня. При плотной низкой облачности эти величины могут отличаться около 10 раз и при листовой поверхности $L=6$ находиться в пределах от 0,1 до 1 гонны на га в день.

Для дальнейшего уточнения такого рода расчетов нужно более подробно изучать световые кривые листьев разных ярусов при нормальных концентрациях $\mathrm{CO}_{2}$, а также уточнить и повторить для разных посевов спектрофотометрические измерения потоков радиации.

\section{Л И Т Е Р А Т У P A}

1. Кринов Е. Л., Спектральная отражательная способность природных образований, Изд. АН СССР, М.-Л., 1947.

2. Sa uberer F. und $\mathrm{Härtel} \mathrm{O.,} \mathrm{Pflanze} \mathrm{und} \mathrm{Strahlung,} \mathrm{Eeipzig,} 1959$.

3. Р абинович Е., Фотосинте 3, т. II, ИИЛ, М., 1953.

4. Кле шн ин А. Ф., Ш ульгин Н. А., ДАН СССР, 125, 1158 (1959).

5. Т а ге е а С. В., Б р ан т А. Б., Биофизика, 4, 308 (1960)

6. III ахов А. А., С танко С. А., Х а занов В. С., Д в конов Ф. С., Ботан. ж., 14, 1681 (1959).

7. Конд р а тьев К. Я., Метеорол. и гидрология, 5, 46 (1960).

8. Шульгин Н. А., Клешнин А. Ф., Подольный В. З., Физиология растений, вып. 2, 7, 141 (1960).

9. D irm ir n, I., Wetter und Leben, 9, H. 3-5, 41 (1957).

10. S al u berer F., Bioklim. Beibl. Met. Zeitschr., 4, 147 (1937).

11. Ал ексеев В. А., Сб. Проблемы экологии и физиологии лесных растений, изд ЛТА, 1963, стр. 47.

12. E g le K., Planta, 26, 546 (1937).

13. Seybold A., J. Wiss. Bot., 82, 741 (1936)

14. Knuchel H., Mitt. Schweiz. Centralanstalt forstl. Versuchswesen, 11, 1 (1914).

15. Ш и фр ин К. С., Тр. ГГО, 39(101), 224 (1953).

16. I s o be S.; Bull. Nat. Inst. Agric. Sci. Nishigahara, Tokyo, 9A, 29 (1962).

17. Р осс Ю. К., Научн. сообщ. ин-та геологии и географии АН Лит. ССР, 13, 15 ! (1962).

18. Росс Ю. Қ., Н ил ьсон Т., Исслед. по физике атмосферы, 4, Тарту, 1963, стр. 42.

19. Т о оминг Х. Г., Днссертация, Тарту, 1961.

20. Ни йлиск Х., Нзв. АН ЭССР, Серия физ.-матем. и техн. наук, №. 1, (1965; (в печати).

21. Нийлиск Херб́ерт, Нсслед. по физике атмосферы, 3, Тарту, 1962, стр. 150.

22. Ннйлиск Херберт, Нсслед. по фнзике атмосферы, 4, Тарту, 1963, стр. 120.

23. Ав а ст е О., Молд а у Х. и Ши фрин К. С., Нсслед. по физике атмосферы, 3. Тарту, 1962, стр. 23.

24. Кондр атьев К. Я., Бургов а М. Л., Гришечкин В. С., Мих айлов В. В., П е т елин Г. М., Сб. Проблемы физики атмосферы, 2, Изд. Лен. унив., 1963, стр. 67

25. Ничилорович А. А., Физиол. растений, выг. $6,7,744$ (1960)

26. Кондр а тьев К. Я., Лучистая энергия Солнца. Гидрометеонздат, Л., 1954, стр. 386.

27. Кринов Л. и Ш аронов В. В., Журнал геофизикн, вып. $2-3,6$ (1936)

28. Schulze R., Ann. d. Meteorol., B. 4, H. 1/6 (1951)

29. Se y b o 1 d A., Bot. Arch., 44, 102 (1942).

30. Verduin J. and L o o m is W. E., Plant Physiol., 19, Nr. 2 (1944).

31. С троганов а Л. Е.. Сб. Фотосннтез и нопросы продуктивности растенин,, Изд. AH CCCP, M., 1963 , стр. 71.

32. Hesketh J. D. and Moss. D. N., Crop Science, 3, № 2, 107 (1963). 


\title{
MAISIKULVI SPEKTRAALNE KIIRGUSREZIIM JA FOTOSUNTEETILISELT AKTIIVSE KIIRGUSE (FAR) ARVUTAMINE
}

\author{
Herbert Niilisk
}

\section{Resümee}

Töös esitałakse kiirgusrežiimi spektrofotomeetriliste mōõtmiste tulemused maisi külvi puhul. Andmed pärinevad autorilt, nad on kogutud 1963. a. suvel Jõgeval põllukultuuride fotosünteesi ja kiirgusrežiimi utrimise komplekssel ekspeditsioonil. Mõõtmisi teostati viiel lainepikkusel: 400,490,552,676 ja $764 \mathrm{mu}$. Suhtelistes ühikutes antakse taimkatte (külvi) sees esinevad spektraalsed kiirgusvood mitmesuguse suhtelise lehepinna puhul (tabelid 1 ja 2). Mōōdetud üles- ia a!lasuunatud voogude kaudu leitakse külvi mitmesugustes kihtides neeldunud kiirgus. Teatud eeldustel jagatakse taimkatte sees mõõdetud kiirgusvood üksikuteks komponentideks (summaarne, otsene, taeva hajus ja taimelehtedelt hajunud kiirgus) (joon. 5)

Töö teises osas antakse fotosünteetiliselt aktiivse kiirguse (FAR) arvutamiseks metoodika, juhuks kui spektraalsed kiirgusvood on mõõdetud üksikutel diskreetsetel lainepikkustel. Esitatud metoodika abil on arvutatud FAR ja toodud FAR-i protsent summaarses kiirguses selge ilma puhul $(17,5-57,5 \%$, keskmine $52,5 \%)$ ning ühtlase madala pilvisuse puhul $(S c, 60 \%)$. Edasi on arvutatud nii läbitulnud kui ka neeldunud FAR taimkatte sees (joon. 10). Kasutades neeldunud FAR-i andmeid ja maisi fotosünteesi valguskōveraid, tuuakse mõned külvi fotosünteesi arvutamise näited.

\section{SPECTRAL RADIATION REGIME OF A MAIZE CROP AND CALCULATION OF THE PHOTOSYNTHETICALLY ACTIVE RADIATION (FAR)}

\author{
Herbert Niilisk
}

\section{Summary}

In the paper results of the spectrophotometric measurements of radiation regime in a maize crop are given. The data presented were obtained by the author in the staff of a complex expedition researching the photosynthesis and radiation regime of the field crops at Jõgeva (Estonian S.S.R.) in summer, 1963. The measurements were carried out at wavelengths $400,490,552,676$ and $764 \mathrm{~m} \mu$. In the paper are presented the relative units of the spectral radiation fluxes in the middle of the plant cover (crop) at various values of the leaf area index (LAI) (tables 1 and 2). On the basis of the measured downward and upward fluxes, the radiation fluxes absorbed in various layers of the plant cover are calculated. According to certain assumptions, the radiation fluxes in the middle of the plant cover are divided into certain components (global, direct solar, diffurse sky and diffused by plant radiation fluxes) (fig. 5)

Iil the second part a method is proposed for calculating the photosynthetically active radiation (FAR) when the spectral radiation fluxes are available only at few waveiengths. According to this method, the percentage of FAR in the global radiation in the case of a clear sky $(47.5-57.5 \%$, the mean value $52.5 \%)$ and in the case of a homogeneous cover of Sc clouds $(60 \%)$ are calculated. The transmitted and absorbed FAR fluxes in the middle of the plant cover are found as well (fig. 10). Using the data on the absorbed FAR and the light curves of photosynthesis some examples of estimating the photosynthesis are presented.

Academy of Sciences of the Estonian S.S.R.,

Institute of Physics and Astronomy
Received April 6th, 1964 